\title{
INFLUÊNCIA DO BRANQUEAMENTO, PASTEURIZAÇÃO E CONGELAMENTO NAS CARACTERÍSTICAS FÍSICO-QUÍMICAS E CAROTENOIDES DE POLPA DE ARATICUM
}

\author{
LETÍCIA LINHARES SILVA ${ }^{1+1 *}$ \\ LEANDRO DE MORAIS CARDOSO ${ }^{2+}$ \\ HELENA MARIA PINHEIRO-SANT'ANA ${ }^{3+}$
}

\begin{abstract}
Neste estudo avaliaram-se os efeitos da pasteurização, branqueamento e congelamento nas características físico-químicas e carotenoides de polpa de araticum. As características físico-químicas $(\mathrm{pH}$, acidez titulável e sólidos solúveis) e carotenoides pró-vitamínicos $\mathrm{A}$ ( $\alpha$-caroteno e $\beta$-caroteno) foram determinados na polpa in natura (controle) e em polpas branqueadas $\left(70^{\circ} \mathrm{C}, 2\right.$ minutos) e pasteurizadas $\left(75^{\circ} \mathrm{C}, 30\right.$ minutos) submetidas a congelamento por 180 dias $\left(-5\right.$ ou $\left.-18^{\circ} \mathrm{C}\right)$. A acidez titulável foi determinada por titulometria, $\mathrm{pH}$ por potenciometria e sólidos solúveis por refratometria. Os carotenoides foram analisados por cromatografia líquida de alta eficiência, com detecção por arranjo de diodos. A polpa in natura apresentou elevado teor de sólidos solúveis $\left(21,27^{\circ} \mathrm{Brix}\right)$, all-trans- $\alpha$-caroteno $(1,98 \mathrm{mg} / 100 \mathrm{~g})$, all-trans- $\beta$ caroteno $(1,58 \mathrm{mg} / 100 \mathrm{~g})$ e valor de vitamina $A(131,46 \mathrm{RAE} / 100 \mathrm{~g})$. As características físico-químicas da polpa não se modificaram após a pasteurização, branqueamento e durante o armazenamento $(p>0,05)$. No entanto, o all-trans- $\alpha$-caroteno, all-trans- $\beta$-caroteno e valor de vitamina $A$ diminuíram após os tratamentos térmicos $(p<0,05)$ (em média, $-18,-41$ e $-41 \%$, respectivamente). Durante o armazenamento, ambas as temperaturas de congelamento influenciaram as polpas de forma semelhante $(p>0,05)$. Os carotenoides e valor de vitamina A nas polpas branqueadas e pasteurizadas congeladas variaram de forma similar até 90 dias de armazenamento. No entanto, após 180 dias, os carotenoides e valor de vitaminaAaumentaram nas polpas pasteurizadas (em média, $+6,5 \%,+33,0 \%$ e $+33,5 \%$, respectivamente) e reduziram nas polpas branqueadas (-23,5\%, $-19,5 \%$ e $-19,5 \%$, respectivamente). Em conclusão, o binômio branqueamento/congelamento mostrou-se eficaz para armazenamento de polpa até 90 dias sendo que, após esse período, recomenda-se o uso da pasteurização/congelamento.
\end{abstract}

PALAVRAS-CHAVE: ANNONA CRASSIFLORA MART.; ESTABILIDADE DE CAROTENOIDES; FRUTO DO CERRADO; POLPA DE FRUTA.

11 Graduanda em Nutrição pela Universidade Federal de Viçosa - UFV (e-mail: leticia.linhares@ ufv.br);

2 Mestre em Ciência da Nutrição pela UFV (e-mail: leandro.cardoso@ufv.br).

${ }^{3}$ Doutora em Ciência dos Alimentos pela Universidade de São Paulo (e-mail: helena.santana@ufv. br).

† Laboratório de Análise de Vitaminas, Departamento de Nutrição e Saúde, Universidade Federal de Viçosa, Viçosa, Minas Gerais

"Endereço para correspondência: Laboratório de Análise de Vitaminas, Departamento de Nutrição e Saúde, CCB II, UFV, Viçosa, Minas Gerais, 36571-000, Brasil. Tel: +55 (31) 3899-1684. Email: leticia.linhares@ufv.br 


\section{INTRODUÇÃO}

O araticum (Annona crassiflora Mart.) é um fruto nativo do Cerrado Brasileiro que possui elevado valor energético, teor de nutrientes e compostos bioativos (Cardoso, Oliveira et al., 2013). A polpa do fruto possui minerais ( $\mathrm{Ca}, \mathrm{Zn}, \mathrm{Cu}, \mathrm{Fe}, \mathrm{Pe} \mathrm{Mg}$ ) e vitaminas (vitamina $\mathrm{C}$, vitamina $\mathrm{E}$ e folatos) (De Souza, Pereira et al., 2012; Cardoso, Oliveira et al., 2013) e destaca-se como excelente fonte de fibras alimentares e carotenoides pró-vitamínicos A (Cardoso, Oliveira et al., 2013).

$\mathrm{O}$ araticum possui elevada massa e rendimento de polpa (Cardoso, Oliveira et al., 2013) e sua polpa apresenta alto aproveitamento culinário e é consumida in natura ou processada na forma de sucos, licores, doces, geleias, tortas e iogurtes (Silva, Silva et al., 2001; Oliveira, Ribeiro et al., 2008). Este fruto constitui uma alternativa para a indústria, uma vez que os produtos derivados de sua polpa possuem boa aceitação e intenção de compra pelo consumidor (Oliveira, Ribeiro et al., 2008; Rocha, Cobucci et al., 2008).

O uso do araticum pela população e indústria de alimentos é restrito devido à curta safra e alta perecibilidade do fruto resultante das suas caracteristicas químicas, tais com o elevado $\mathrm{pH}$ que favorece o desenvolvimento de microoganismos (Ramos, Benevides et al., 2010; Cardoso, Oliveira et al., 2013). Neste contexto, a produção de polpa de araticum constitui uma importante alternativa para permitir a sua estocagem na entressafra dos frutos.

A polpa de fruta é definida como produto não fermentado, não concentrado ou diluído, obtido pelo esmagamento de frutos carnosos. A legislação brasileira estabelece padrões microbiológicos, de identidade e características físico-químicas mínimas para alguns tipos de polpa (Brasil, 1978; 2000; 2001).

Diversos métodos podem ser utilizados para a conservação de polpa de frutas, entre os quais se destacam as técnicas com emprego ou remoção de calor (branquamento, pasteurização e congelamento). Esses métodos inibem o crescimento de microrganismos e a atividade enzimática e, dessa forma, aumentam a vida de prateleira e a segurança microbiológica dos produtos (ElezMartínez e Martín-Belloso, 2007; Fellows, 2008). No entanto, podem alterar o teor de alguns nutrientes como as vitaminas e carotenoides.

Os carotenoides são compostos insaturados altamente susceptíveis à ação da luz, temperatura entre outros (Ambrósio, Campos et al., 2006). Alguns carotenoides, como o a-caroteno, $\beta$-caroteno e $\beta$-criptoxantina, são convertidos no organismo em vitamina A (Rodriguez-Amaya e Kimura, 2004) a qual é essencial para a diferenciação celular, a visão, o crescimento ósseo, a reprodução e a integração do sistema imunológico (Williams, Boileau et al., 2000; Mursu, Nurmi et al., 2008). Além disso, os carotenoides contribuem para a prevenção e o tratamento de doenças cardiovasculares e a modulação do câncer (Stahl e Sies, 2002; Tapiero, Townsend et al., 2004; Krinsky e Johnson, 2005; Mursu, Nurmi et al., 2008).

Uma vez que os consumidores têm se tornado cada vez mais exigentes e optado por produtos com maior vida de prateleira e que mantenham suas características sensoriais e nutricionais durante o armazenamento (Rivas, Rodrigo et al., 2006), o objetivo deste estudo foi avaliar o impacto da pasteurização, branqueamento e congelamento $\left(-5^{\circ} \mathrm{C}\right.$ e $\left.-18^{\circ} \mathrm{C}\right)$ nas características físicoquímicas e no teor de carotenoides de polpa de araticum (Annona crassiflora Mart.).

\section{MATERIAL E MÉTODOS}

\subsection{COLETA, AMOSTRAGEM E SELEÇÃO DOS FRUTOS}

Frutos do araticum (Annona crassiflora Mart.) nativos do Cerrado Brasileiro (Município de Curvelo, Minas Gerais) foram coletados em época de safra (fevereiro a março de 2011), após 
sua queda natural da árvore. A amostragem foi realizada em 3 repetições sendo cada uma delas composta por $2,5 \mathrm{~kg}$ de frutos ( \pm 5 unidades) coletados em áreas e árvores distintas.

Após a coleta, os frutos foram acondicionados em caixas de isopor e transportados para o laboratório, em até 36 horas. No laboratório, os frutos morfologicamente perfeitos e com sólidos solúveis superiores a $19^{\circ}$ Brix (maturação fisiológica completa) foram selecionados para análise.

\subsection{OBTENÇÃO E PROCESSAMENTO DA POLPA}

A casca, a polpa e as sementes do araticum foram separadas com auxílio de uma faca em inox. A polpa foi homogeneizada em processador doméstico de alimentos (Faet Multipratic, MC5), acondicionada em sacos de polietileno $(30 \mathrm{~g})$, submetida ao tratamento térmico (pasteurização ou branqueamento) e congelada por 180 dias $\left(-5^{\circ} \mathrm{C}\right.$ e $\left.-18^{\circ} \mathrm{C}\right)$ (Figura 1).

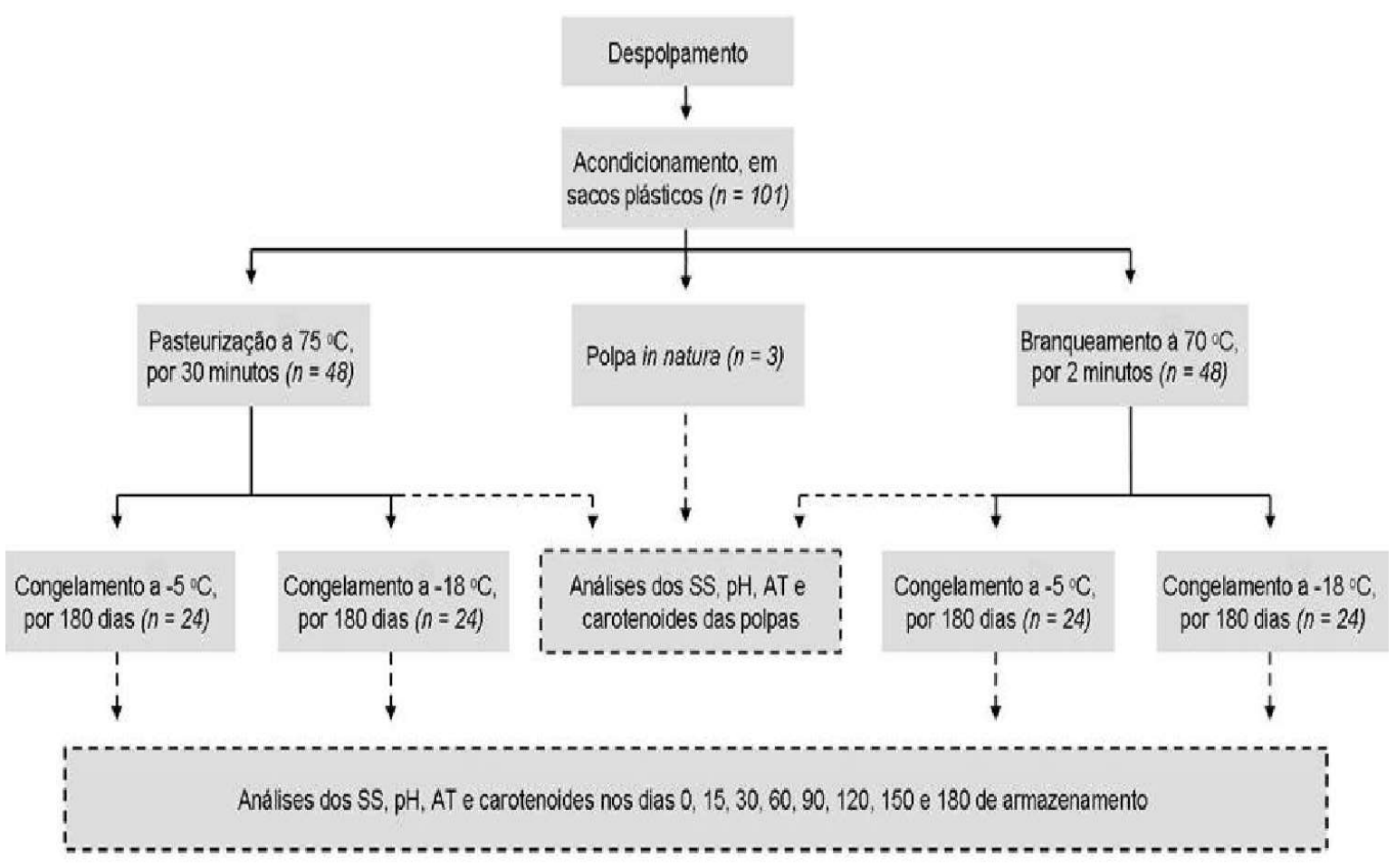

FIGURA 1: FLUXOGRAMA DE PROCESSAMENTO E ANÁLISE DA POLPA DE ARATICUM $\mathrm{n}$ = número de parcelas (sacos plásticos) submetidas ao tratamento.

\subsection{ANÁLISES QUÍMICAS}

Para as análises físico-químicas, extração e obtenção de padrões de carotenoides foram utilizados reagentes grau analítico. Nas análises de carotenoides foram utilizados os seguintes reagentes grau HPLC: acetona, acetato de etila, metanol e acetonitrila (Tedia, Brasil). As análises físico-químicas e de carotenoides foram realizadas antes e após o tratamento térmico (T0) e durante o congelamento $(15,30,60,90,120,150$ e 180 dias $)$.

\subsubsection{Análises Físico-Químicas}

A acidez titulável, pH e sólidos solúveis da polpa de araticum foram avaliados de acordo com o Instituto Adolfo Lutz (2005). A acidez titulável (AT) foi determinada por volumetria de neutralização utilizando solução padrão de hidróxido de sódio $0,1 \mathrm{~mol} / \mathrm{L}$. $\mathrm{O} \mathrm{pH}$ foi determinado diretamente na polpa utilizando pHmetro digital de bancada (Digimed, DM-22), e os sólidos solúveis (SS) foram determinados por refratometria utilizando refratômetro óptico (Analytik Jena). 


\subsubsection{Análise de Carotenoides}

Foram investigados a presença e teor de all-trans- $\alpha$-caroteno, all-trans- $\beta$-caroteno, all-translicopeno e all-trans- $\beta$-criptoxantina na polpa de araticum utilizando os métodos de extração e análise propostos por Kimura e Rodriguez-Amaya (2002) e Pinheiro-Sant'Ana, Stringheta et al. (1998), respectivamente. Cerca de $5 \mathrm{~g}$ de polpa foram adicionados de $20 \mathrm{~mL}$ de acetona resfriada, homogeneizados em microtriturador (Marconi, MA 102) e filtrados a vácuo em funil de buchnner, utilizando-se papel de filtro. O procedimento de extração foi repetido por mais duas vezes no resíduo até a descoloração completa da polpa.

Posteriormente, o filtrado foi transferido em três frações para um funil de separação contendo $50,0 \mathrm{~mL}$ de éter de petróleo. Após a transferência de cada fração, água destilada foi adicionada para a separação de fases (superior: carotenoides em éter de petróleo; inferior: acetonaágua) sendo a fase inferior descartada. Acrescentou-se sulfato de sódio anidro ao extrato etéreo para retirar o resíduo de água. Posteriormente, o extrato foi concentrado em evaporador rotativo (Tecnal, TE-211) a $35 \pm 1{ }^{\circ} \mathrm{C}$ e o volume completado para $25,0 \mathrm{~mL}$ com éter de petróleo. $\mathrm{O}$ extrato foi armazenado em frasco de vidro âmbar hermeticamente vedado e acondicionado a $-18 \pm$ $1^{\circ} \mathrm{C}$, até o momento da análise.

Antes da análise, $8,0 \mathrm{~mL}$ do extrato foram evaporados sob fluxo nitrogênio gasoso e o resíduo seco foi redissolvido em $2 \mathrm{~mL}$ de acetona grau HPLC. Para análise, $50 \mu \mathrm{L}$ de extrato previamente filtrado em unidade filtrante (Millipore, Brasil) com porosidade de 0,45 $\mu \mathrm{m}$ foram injetados na coluna cromatográfica. As análises foram realizadas utilizando um sistema de cromatografia líquida de alta eficiência-CLAE (Shimadzu, SCL 10at VP) composto por bomba de alta pressão (Shimadzu, LC-10at VP), amostrador automático com loop de 50 $\mathrm{mL}$ (Shimadzu, SIL-10AF) e detector de arranjo de diodos (DAD) (Shimadzu, SPD-M10A). Utilizou-se coluna cromatográfica RP18 (Phenomenex Gemini, 250 x 4,6 mm, $5 \mu \mathrm{m}$ ), munida de coluna de guarda (Phenomenex ODS, $4 \mathrm{~mm} \times 3 \mathrm{~mm}$ ); fase móvel composta de metanol: acetato de etila: acetonitrila (80:10:10, v/v/v), fluxo da fase móvel de $2,0 \mathrm{~mL} \mathrm{~min}^{-1}$ e tempo de corrida de 13 minutos (Pinheiro-Sant'Ana, Stringheta et al., 1998).

Durante a extração e análise, as amostras e os extratos foram protegidos da luz (solar e artificial) utilizando vidrarias âmbar, papel alumínio e cortinas do tipo blackout, e protegidos do oxigênio utilizando tampas e ambiente com nitrogênio gás nas vidrarias.

A identificação e quantificação de compostos foram realizadas utilizando $\alpha$-caroteno e $\beta$-caroteno isolados de cenoura e $\beta$-criptoxantina e licopeno isolados de papaia e tomate, respectivamente, por meio de cromatografia em coluna aberta (Kimura e Rodriguez-Amaya, 2002). A pureza dos padrões, obtida em espectrofotômetro (Thermo Scientific, Evolution 60S), foi

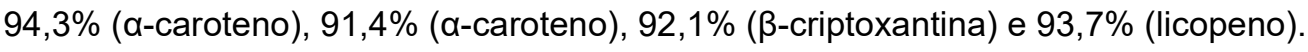

Os compostos foram identificados comparando os tempos de retenção e espectros de absorção obtidos para os padrões e para as amostras analisados sob as mesmas condições. Para a quantificação dos carotenoides encontrados nas amostras foram utilizadas curvas analíticas e equações de regressão construídas por meio da injeção, em duplicata, de seis soluções dos padrões diferentes concentrações (all-trans- $\alpha$-caroteno: 0,033 a 2,060 $\mu \mathrm{g} ; \mathrm{y}=1730130,16 \mathrm{x}-8057,58 ; \mathrm{R}^{2}=$ 0,999; all-trans- $\beta$-caroteno: 0,004 a 1,433 $\mu \mathrm{g} ; \mathrm{y}=1389460,94 \mathrm{x}+24320,87 ; \mathrm{R}^{2}=0,996$ ).

$O$ valor de vitamina $A$ foi estimado utilizando os fatores de conversão do U. S Institute of Medicine (2001) em que 1 Equivalente de Atividade de Retinol (RAE) corresponde a $1 \mu \mathrm{g}$ de retinol; $12 \mu \mathrm{g}$ de $\beta$-caroteno e $24 \mu \mathrm{g}$ de outros carotenoides pró-vitamínicos.

\subsection{DELINEAMENTO EXPERIMENTAL E ANÁLISE DOS DADOS}

Foi utilizado o delineamento inteiramente casualizado, em parcelas subdivididas, com 3 repetições. Os tratamentos térmicos (pasteurização e branqueamento) constituíram as parcelas e 
as temperaturas de armazenamento $\left(-5^{\circ} \mathrm{C} \mathrm{e}-18^{\circ} \mathrm{C}\right)$ representaram as sub-parcelas.

Os dados foram submetidos ao teste de normalidade (Shapiro-Wilk) e a diferença entre os tratamentos avaliada pela análise de variância (ANOVA). As diferenças entre as médias dos tratamentos térmicos (branqueamento e pasteurização) dentro de um mesmo tempo de armazenamento foram avaliadas pelo teste de Tukey $(p<0,05)$. A variação dos dados dentro de um mesmo tratamento térmico ao longo do armazenamento foi avaliada por análise de regressão. A diferença entre os tempos 0 (T0) e 180 (T180) foi analisada pelo teste $t$ de student $(p<0,05)$. As análises estatísticas foram realizadas utilizando-se o software SAS (Statistical Analisys System), versão 9.2 (2008), licenciado para a UFV.

\section{RESULTADOS E DISCUSSÃO}

\subsection{CARACTERÍSTICAS FÍSICO-QUÍMICAS E CAROTENOIDES DA POLPA DE ARATICUM IN NATURA}

Até o momento, não existe uma Instrução Normativa Brasileira que estabelece os padrões de identidade e qualidade para polpa de araticum. A polpa de araticum in natura apresentou sólidos solúveis, $\mathrm{pH}$ e acidez titulável superiores aos observados em frutos coletados no Norte de Minas Gerais (De Souza, Pereira et al., 2012) (Tabela 1). Essas diferenças podem se dever ao fato de os frutos analisados nestes estudos terem sido obtidos a partir de árvores nativas em estado selvagem, o que contribui para a variação nas características químicas dos frutos.

A polpa de araticum in natura apresentou all-trans- $\alpha$-caroteno (tempo de retenção - TR: 11,2 minutos) e all-trans- $\beta$-caroteno (TR: 11,8 minutos) (Figura 2). Diferente do observado por outros autores (De Souza, Pereira et al., 2012; Cardoso, Oliveira et al., 2013), o all-trans-licopeno não foi identificado na polpa de araticum. A variação no perfil de carotenoides em alimentos é pouco comum, no entanto, pode ser observada em algumas variedades de frutas e hortaliças tais como abóbora, pimenta, ameixa (Rodriguez-Amaya, 1999).

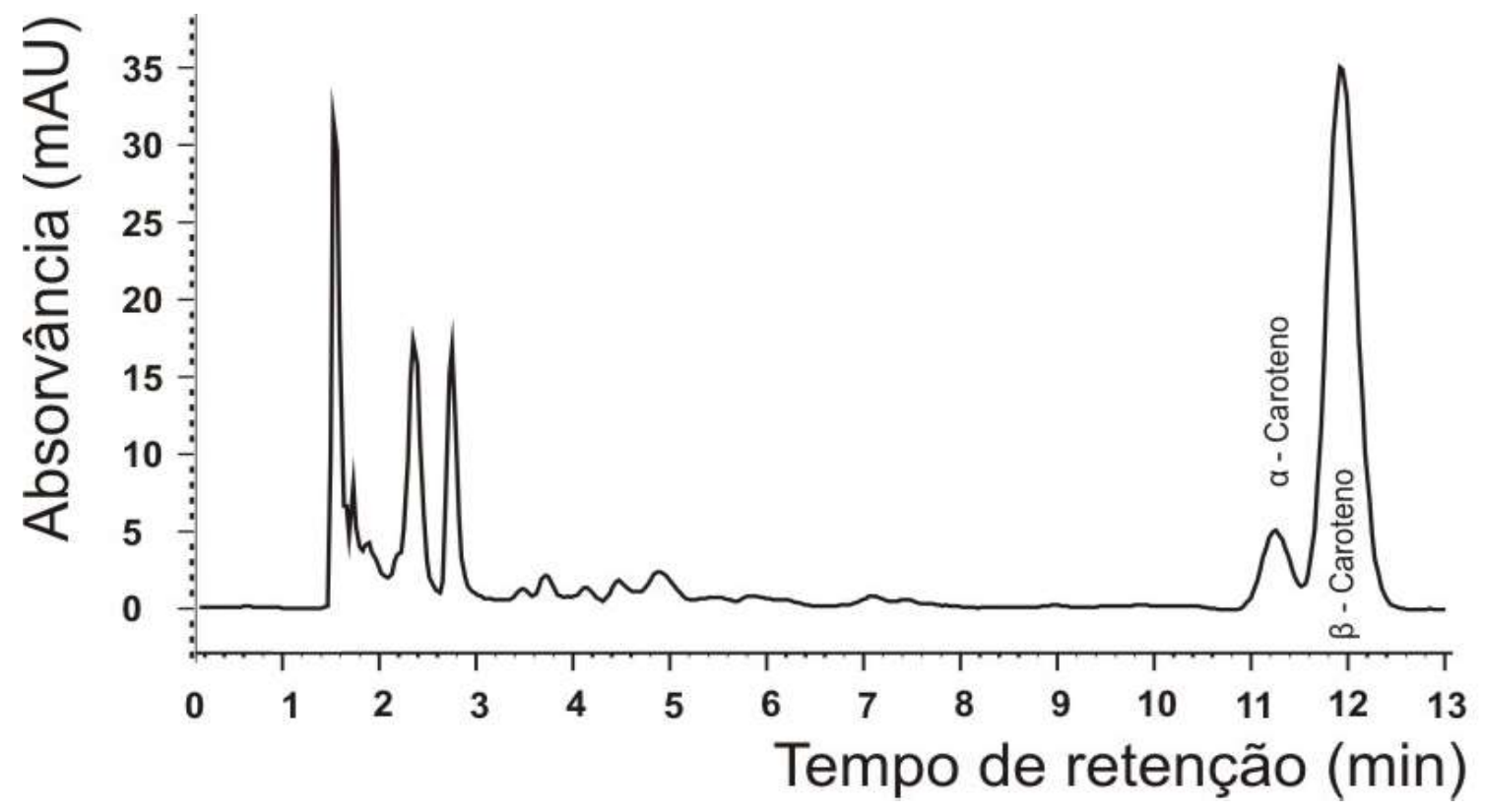

\section{FIGURA 2: ANÁLISE POR CLAE DE CAROTENOIDES EM POLPA DE ARATICUM (ANNONA CRASSIFLORA MART.) DO CERRADO (CURVELO, MINAS GERAIS, BRASIL).}

A polpa de araticum apresentou elevado teor de all-trans- $\alpha$-caroteno, all-trans- $\beta$-caroteno, totais de carotenoides e valor de vitamina A (Tabela 1). No entanto, esses valores foram de 20 a 
$55 \%$ menores aos previamente obtidos em nosso laboratório em frutos coletados na mesma região (all-trans- $\alpha$-caroteno: $2,98 \mathrm{mg} / 100 \mathrm{~g}$; all-trans- $\beta$-caroteno: $1,97 \mathrm{mg} / 100 \mathrm{~g}$; totais de carotenoides: $4,98 \mathrm{mg} / 100 \mathrm{~g}$ e valor de vitamina A: 288,79 RAE/100g) (Cardoso, Oliveira et al., 2013). Essas diferenças sugerem que o teor de carotenoides no araticum também pode ser influenciado por fatores relacionados ao ano da safra, condições climáticas e condições fisiológicas da árvore.

\subsection{EFEITO DO TRATAMENTO TÉRMICO NAS CARACTERÍSTICAS FÍSICO-QUÍMICAS E CAROTENOIDES}

Apesar da diferença no binômio tempo/temperatura dos tratamentos térmicos, os efeitos do branqueamento e pasteurização nas características físico-químicas e carotenoides da polpa foram semelhantes (Tabela 1).

\section{TABELA 1: IMPACTO DO TRATAMENTO TÉRMICO NAS CARACTERÍSTICAS FÍSICO- QUÍMICAS, TEOR DE CAROTENOIDES E VALOR DE VITAMINA A DA POLPA DE ARATICUM.}

\begin{tabular}{|c|c|c|c|}
\hline Variáveis & In natura & Branqueamento & Pasteurização \\
\hline Acidez titulável (g ácido cítrico/100g) & $0,60 \pm 0,11^{\mathrm{a}}$ & $0,52 \pm 0,10^{\mathrm{a}}$ & $0,49 \pm 0,07^{\mathrm{a}}$ \\
\hline $\mathrm{pH}$ & $4,71 \pm 0,03^{a}$ & $4,72 \pm 0,06^{\mathrm{a}}$ & $4,77 \pm 0,01^{\mathrm{a}}$ \\
\hline Sólidos solúveis ( ${ }^{\circ}$ Brix) & $21,27 \pm 1,67^{\mathrm{a}}$ & $21,67 \pm 0,61^{a}$ & $21,00 \pm 2,36^{\mathrm{a}}$ \\
\hline all-trans- $\alpha$-caroteno $(\mathrm{mg} / 100 \mathrm{~g})$ & $1,98 \pm 0,07^{\mathrm{a}}$ & $1,71 \pm 0,09^{b}$ & $1,55 \pm 0,10^{b}$ \\
\hline $\begin{array}{l}\text { all-trans- } \beta \text {-caroteno }(\mathrm{mg} / 100 \mathrm{~g}) \\
\text { Total de carotenoides }(\mathrm{mg} / 100 \mathrm{~g})\end{array}$ & $\begin{array}{l}1,58 \pm 0,14^{\mathrm{a}} \\
3,55 \pm 0,20^{\mathrm{a}}\end{array}$ & $\begin{array}{l}1,01 \pm 0,09^{\mathrm{b}} \\
2,76 \pm 0,26^{\mathrm{b}}\end{array}$ & $\begin{array}{l}0,86 \pm 0,06^{b} \\
2,41 \pm 0,15^{b}\end{array}$ \\
\hline Valor de vitamina A (RAE/100g) & $131,46 \pm 11,68^{a}$ & $84,57 \pm 7,82^{b}$ & $71,45 \pm 4,74^{b}$ \\
\hline
\end{tabular}

${ }^{1}$ Valores expressos em matéria fresca; ${ }^{2}$ média de 3 repetições; ${ }^{3}$ dados apresentados em média \pm desvio padrão; ${ }^{4}$ Médias seguidas de uma mesma letra na linha não diferem entre si pelo teste de Tukey ao nível de $5 \%$ de significância.

Os tratamentos térmicos não alteraram as características físico-químicas da polpa, mas reduziram o teor de all-trans- $\alpha$-caroteno (em média, -18\%), all-trans- $\beta$-caroteno (em média, $-41 \%$ ) e, consequentemente, os carotenoides totais e valor de vitamina A (em média, -27 e $-41 \%$, respectivamente). A redução no teor dos all-trans-carotenoides pode ser atribuída à isomerização destes compostos às formas cis, devido à ação do calor (Pénicaud, Achir et al., 2011), diminuindo a atividade biológica dos carotenoides e o valor nutricional do alimento (Rodriguez-Amaya, 1999).

\subsection{ESTABILIDADE AO LONGO DO ARMAZENAMENTO}

As características físico-químicas e os carotenoides nas polpas de araticum variaram em função do tipo de tratamento térmico $(p<0,05)$, mas não foram influenciadas pela temperatura de congelamento e pela interação destas variáveis $(p>0,05)$ (Tabela 2, Figura 3 ). Dessa forma, as polpas submetidas a um mesmo tratamento térmico apresentaram comportamento semelhante durante todo o período de armazenamento.

\subsubsection{Características Físico-Químicas}

OpHe a acidez titulável das polpas branqueadas e pasteurizadas submetidas ao congelamento não variaram durante o armazenamento (Tabela 2). A ausência de variação nestes parâmetros está de acordo com o observado em polpas de acerola (Lima, Figueiredo et al., 2012). Além disso, os sólidos solúveis nas polpas pasteurizadas e branqueadas também se mantiveram constantes durante 
o armazenamento, o que sugere uma excelente estabilidade dos compostos solúveis presentes na polpa de araticum. O comportamento dos sólidos solúveis em polpas de frutas é bastante heterogêneo e parece ser influenciado pelo perfil de compostos solúveis (pectina, açucares redutores, vitaminas, entre outros) da matriz alimentar (Lima, Figueiredo et al., 2012). Em estudos recentes, foi observado decréscimo ou aumento no teor destes compostos em polpas tratadas em condições similares às do presente estudo (Lima, Figueiredo et al., 2012; Damiani, Lage et al., 2013).

\subsubsection{Carotenoides e Valor de Vitamina A}

As alterações do teor de carotenoides durante o armazenamento decorrem de processos complexos os quais são influenciados por diversos fatores e afetam a concentração desses compostos de forma inconsistente (Lin e Chen, 2005). No presente estudo, o teor de $\alpha$-caroteno, $\beta$-caroteno, total de carotenoides e valor de vitamina $A$ das polpas não foi influenciado pela temperatura de armazenamento e diferiram entre os tratamentos térmicos a partir de 60 dias de armazenamento (Figura 3).

O teor de all-trans- $\alpha$-caroteno, all-trans- $\beta$-caroteno nos quatro tipos de polpas congeladas reduziu até o $30^{\circ}$ dia de armazenamento. Esta redução pode ser resultado da isomerização transcis, oxidação e degradação dos carotenoides (Zepka, Borsarelli et al., 2009; Pénicaud, Achir et al., 2011). Após 30 dias de armazenamento, verificou-se aumento no teor de carotenoides, o que demonstrou a ocorrência de uma fase de balanço positivo onde a conversão de outros carotenoides a all-trans- $\alpha$-caroteno e all-trans- $\beta$-caroteno superou as reações de degradação (Zepka, Borsarelli et al., 2009; Pénicaud, Achir et al., 2011).
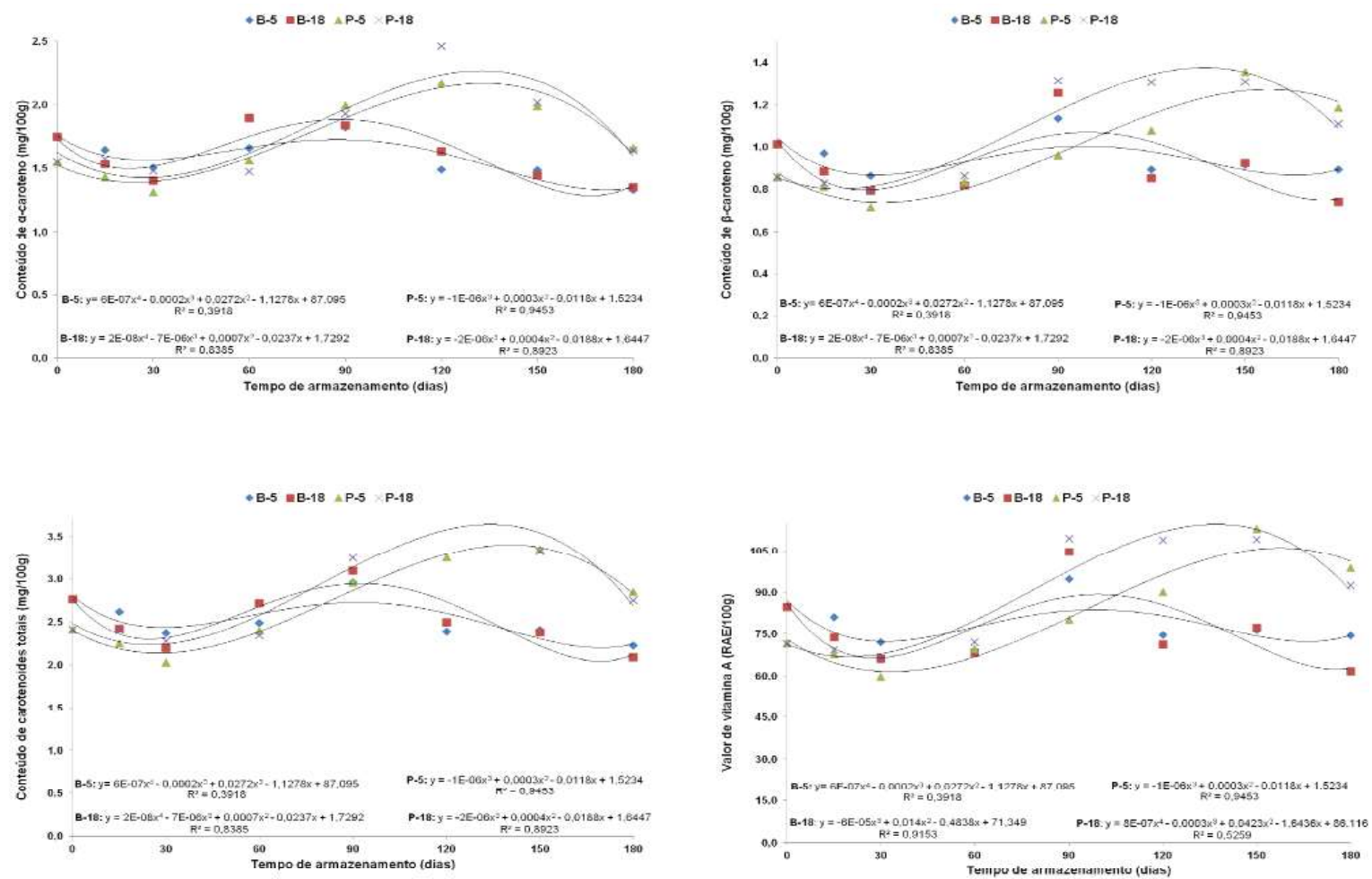

FIGURA 3: COMPORTAMENTO E EQUAÇÃO DE REGRESSÃO AJUSTADA PARA

ALL-TRANS-A-CAROTENO (A), ALL-TRANS-B-CAROTENO (B), TOTAL DE CAROTENOIDES (C) E VALOR DE VITAMINA A (D) EM POLPAS DE ARATICUM SUBMETIDAS A TRATAMENTO TÉRMICO (PASTEURIZAÇÃO OU BRANQUEAMENTO) E CONGELAMENTO $\left(-5^{\circ} \mathrm{C}\right.$ OU $\left.-18^{\circ} \mathrm{C}\right)$ POR 180 DIAS

B-5: polpa branqueada congelada a $-5^{\circ} \mathrm{C}$; B-18: Polpa branqueada congelada a $-18^{\circ} \mathrm{C}$; P-5: polpa pasteurizada congelada a $-5^{\circ} \mathrm{C}$; P-18: Polpa pasteurizada congelada a $-18^{\circ} \mathrm{C}$; 
TABELA 2: SÓLIDOS SOLÚVEIS, PH E ACIDEZ TITULÁVEL EM POLPA DE ARATICUM PASTEURIZADAS OU BRANQUEADAS, E CONGELADAS $\left(-5^{\circ} \mathrm{C}\right.$ OU $\left.-18^{\circ} \mathrm{C}\right)$, POR 180 DIAS

$1,2,3$

\begin{tabular}{|c|c|c|c|c|}
\hline \multicolumn{5}{|c|}{ Sólidos Solúveis ( $\left.{ }^{\circ} \mathrm{Brix}\right)$} \\
\hline Tempo & Branqueada $\left(-5^{\circ} \mathrm{C}\right)$ & Branqueada $\left(-18^{\circ} \mathrm{C}\right)$ & Pasteurizada $\left(-5^{\circ} \mathrm{C}\right)$ & Pasteurizada $\left(-18^{\circ} \mathrm{C}\right)$ \\
\hline 0 & $21,67 \pm 0,61^{\mathrm{Aa}}$ & $21,67 \pm 0,61^{\mathrm{Aa}}$ & $21,00 \pm 2,36^{\mathrm{Aa}}$ & $21,00 \pm 2,36^{\mathrm{Aa}}$ \\
\hline 15 & $20,27 \pm 0,63^{\mathrm{Aa}}$ & $20,80 \pm 0,60$ Аа & $21,50 \pm 1,31^{\mathrm{Aa}}$ & $21,40 \pm 1,11^{\mathrm{Aa}}$ \\
\hline 30 & $21,63 \pm 0,76^{\mathrm{Aa}}$ & $20,70 \pm 0,78^{\mathrm{Aa}}$ & $21,20 \pm 1,31^{\mathrm{Aa}}$ & $21,07 \pm 1,10^{\mathrm{Aa}}$ \\
\hline 60 & $21,63 \pm 0,47^{\mathrm{Aa}}$ & $21,43 \pm 0,42^{\mathrm{Aa}}$ & $21,33 \pm 0,96^{\mathrm{Aa}}$ & $22,03 \pm 1,85^{\mathrm{Aa}}$ \\
\hline 90 & $20,73 \pm 0,85^{\mathrm{Aa}}$ & $20,83 \pm 0,35^{\mathrm{Aa}}$ & $22,00 \pm 0,30^{\mathrm{Aa}}$ & $21,77 \pm 0,75^{\mathrm{Aa}}$ \\
\hline 120 & $21,43 \pm 0,35^{\mathrm{Aa}}$ & $21,90 \pm 0,36^{\text {Аa }}$ & $21,87 \pm 1,21^{\mathrm{Aa}}$ & $21,47 \pm 1,86^{\mathrm{Aa}}$ \\
\hline 150 & $21,43 \pm 0,35^{\mathrm{Aa}}$ & $21,73 \pm 0,40^{\mathrm{Aa}}$ & $22,87 \pm 1,21^{\mathrm{Aa}}$ & $21,63 \pm 1,11^{\mathrm{Aa}}$ \\
\hline 180 & $20,63 \pm 0,61^{\mathrm{Aa}}$ & $20,27 \pm 1,01 \mathrm{Aa}$ & $22,65 \pm 1,75^{\mathrm{Aa}}$ & $22,05 \pm 1,25^{\mathrm{Aa}}$ \\
\hline \multicolumn{5}{|c|}{ Acidez titulável (g ácido cítrico/100g) } \\
\hline Tempo & Branqueada $\left(-5^{\circ} \mathrm{C}\right)$ & Branqueada $\left(-18^{\circ} \mathrm{C}\right)$ & Pasteurizada $\left(-5^{\circ} \mathrm{C}\right)$ & Pasteurizada $\left(-18^{\circ} \mathrm{C}\right)$ \\
\hline 0 & $0,52 \pm 0,10^{\mathrm{Aa}}$ & $0,52 \pm 0,10^{\mathrm{Aa}}$ & $0,49 \pm 0,07^{\mathrm{Aa}}$ & $0,49 \pm 0,07^{\mathrm{Aa}}$ \\
\hline 15 & $0,49 \pm 0,07^{\mathrm{Aa}}$ & $0,48 \pm 0,07^{\text {Аa }}$ & $0,49 \pm 0,03^{\mathrm{Aa}}$ & $0,51 \pm 0,03^{\mathrm{Aa}}$ \\
\hline 30 & $0,52 \pm 0,07^{\mathrm{Aa}}$ & $0,53 \pm 0,06^{\mathrm{Aa}}$ & $0,52 \pm 0,05^{\mathrm{Aa}}$ & $0,52 \pm 0,04^{\mathrm{Aa}}$ \\
\hline 60 & $0,52 \pm 0,03^{\mathrm{Aa}}$ & $0,52 \pm 0,03^{\text {Аa }}$ & $0,51 \pm 0,03^{\mathrm{Aa}}$ & $0,53 \pm 0,04^{\mathrm{Aa}}$ \\
\hline 90 & $0,51 \pm 0,06^{\mathrm{Aa}}$ & $0,50 \pm 0,06^{\mathrm{Aa}}$ & $0,49 \pm 0,02^{\mathrm{Aa}}$ & $0,52 \pm 0,02^{\mathrm{Aa}}$ \\
\hline 120 & $0,50 \pm 0,04^{\mathrm{Aa}}$ & $0,50 \pm 0,03^{\mathrm{Aa}}$ & $0,49 \pm 0,03^{\mathrm{Aa}}$ & $0,49 \pm 0,01^{\mathrm{Aa}}$ \\
\hline 150 & $0,52 \pm 0,08^{\mathrm{Aa}}$ & $0,51 \pm 0,08^{\mathrm{Aa}}$ & $0,50 \pm 0,03^{\mathrm{Aa}}$ & $0,53 \pm 0,04^{\mathrm{Aa}}$ \\
\hline 180 & $0,49 \pm 0,07^{\mathrm{Aa}}$ & $0,51 \pm 0,08^{\mathrm{Aa}}$ & $0,50 \pm 0,07^{\mathrm{Aa}}$ & $0,51 \pm 0,04^{\mathrm{Aa}}$ \\
\hline \multicolumn{5}{|c|}{$p H$} \\
\hline Tempo & Branqueada $\left(-5^{\circ} \mathrm{C}\right)$ & Branqueada $\left(-18^{\circ} \mathrm{C}\right)$ & Pasteurizada $\left(-5^{\circ} \mathrm{C}\right)$ & Pasteurizada $\left(-18^{\circ} \mathrm{C}\right)$ \\
\hline 0 & $4,72 \pm 0,06^{\mathrm{Aa}}$ & $4,72 \pm 0,06^{\mathrm{Aa}}$ & $4,77 \pm 0,01^{\mathrm{Aa}}$ & $4,77 \pm 0,01^{\mathrm{Aa}}$ \\
\hline 15 & $4,76 \pm 0,05^{\mathrm{Aa}}$ & $4,75 \pm 0,03^{\mathrm{Aa}}$ & $4,73 \pm 0,06^{\mathrm{Aa}}$ & $4,76 \pm 0,03^{\mathrm{Aa}}$ \\
\hline 30 & $4,74 \pm 0,04^{\mathrm{Ab}}$ & $4,74 \pm 0,04^{\mathrm{Ab}}$ & $4,76 \pm 0,02^{\mathrm{Aa}}$ & $4,78 \pm 0,01^{\mathrm{Aa}}$ \\
\hline 60 & $4,71 \pm 0,01^{\mathrm{Ab}}$ & $4,71 \pm 0,05^{\mathrm{Aa}}$ & $4,77 \pm 0,04^{\mathrm{Aab}}$ & $4,75 \pm 0,02^{\mathrm{Aab}}$ \\
\hline 90 & $4,74 \pm 0,10^{\mathrm{Aab}}$ & $4,78 \pm 0,04^{\mathrm{Aa}}$ & $4,78 \pm 0,02^{\mathrm{Ab}}$ & $4,75 \pm 0,03^{\mathrm{Aa}}$ \\
\hline 120 & $4,74 \pm 0,01^{\mathrm{Aa}}$ & $4,72 \pm 0,03^{\mathrm{Aa}}$ & $4,73 \pm 0,01^{\mathrm{Aa}}$ & $4,71 \pm 0,07^{\mathrm{Aa}}$ \\
\hline 150 & $4,73 \pm 0,03^{\mathrm{Aa}}$ & $4,71 \pm 0,02^{\mathrm{Aa}}$ & $4,73 \pm 0,03^{\mathrm{Aa}}$ & $4,72 \pm 0,06^{\mathrm{Aa}}$ \\
\hline 180 & $4,72 \pm 0,05^{\mathrm{Aa}}$ & $4,72 \pm 0,09^{\mathrm{Aa}}$ & $4,70 \pm 0,04^{\mathrm{Aa}}$ & $4,73 \pm 0,07^{\mathrm{Aa}}$ \\
\hline
\end{tabular}

${ }^{1}$ Análises realizadas em 3 repetições; ${ }^{2}$ dados apresentados em média \pm DP; ${ }^{3}$ Médias seguidas por uma mesma letra minúscula na linha e maiúscula na coluna, não diferem entre si pelo teste de Tukey $(p<0,05)$.

No entanto, o aumento do teor de carotenoides nas polpas branqueadas após o T30 se estendeu até o T90, enquanto que nas polpas pasteurizadas ele se prolongou até o T120. O prolongamento do aumento do teor nas polpas pasteurizadas indicou que a pasteurização foi mais eficaz que o branqueamento na inibição da velocidade das reações de degradação e, consequentemente, o retorno à fase de balanço negativo e declínio do teor dos carotenoides. $\mathrm{A}$ menor eficiência do branqueamento pode se justificar pela não inativação de todas as enzimas presentes no alimento (Elez-Martínez e Martín-Belloso, 2007; Fellows, 2008). Dessa forma, 
estas enzimas, mesmo com atividade inibida pelo frio, podem apresentar atividade residual e, assim, provocar alterações químicas durante o armazenamento. Após o T90 nas polpas branqueadas e T120 nas polpas pasteurizadas observou-se um segundo ciclo de redução no teor de carotenoides.

Após 180 dias de armazenamento, o teor de carotenoides e valor de vitamina A em todos os tratamentos variaram significativamente em relação T0 (polpa não congelada) (Figura 4). $O$ teor de carotenoides e valor de vitamina A nas polpas pasteurizadas no tempo 180 foi significativamente maior que no T0 (em média, 6,5\%, 33,0\%, 10,0\% e 33,5\%, respectivamente). Em contrapartida, nas polpas branqueadas esses parâmetros reduziram em média $23,5 \%, 19,5 \%, 22,0 \%$ e 19,5\%, respectivamente.

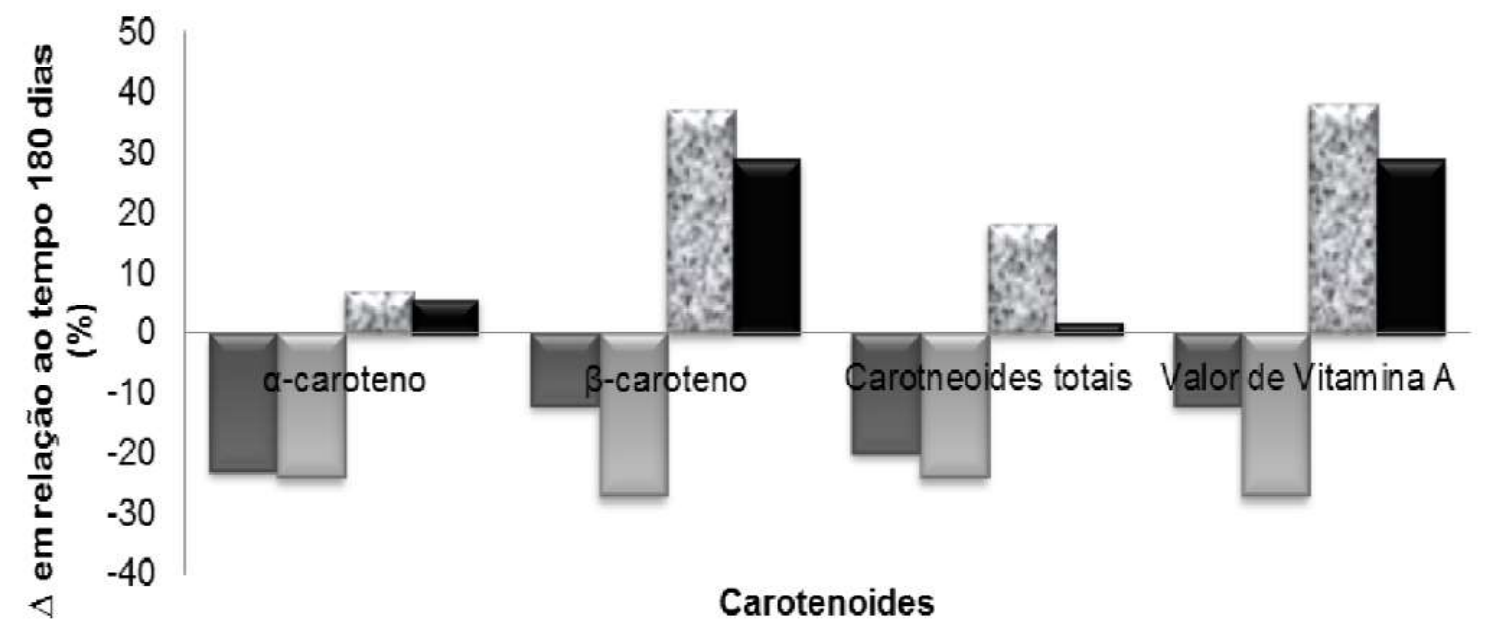

\author{
घranqueada $\left(-5^{\circ} \mathrm{C}\right) \square$ Branqueada $\left(-18^{\circ} \mathrm{C}\right)$ 日Pasteurizada $\left(-5^{\circ} \mathrm{C}\right)$ घPasteurizada $\left(-18^{\circ} \mathrm{C}\right)$ \\ FIGURA 4: VARIAÇÃO ENTRE O TEOR INICIAL (T0) E TEOR FINAL (T180) DE \\ CAROTENOIDES E VALOR DE VITAMINA A EM POLPAS DE ARATICUM SUBMETIDAS A \\ TRATAMENTO TÉRMICO (PASTEURIZAÇÃO OU BRANQUEAMENTO) E CONGELADAS A \\ $-5^{\circ} \mathrm{C}$ OU $-18^{\circ} \mathrm{C}$.
}

O maior teor de carotenoides nas polpas pasteurizadas no tempo 180, em relação ao T0, refletiu o prolongamento da fase de balanço positivo entre os tempos 90 e 120. Este prolongamento retardou o retorno à fase de declínio permitindo que essas polpas, no tempo 180, não se encontrassem em seu balanço negativo máximo, no qual seria possível observar teor de carotenoides inferiores a T0. Nas polpas branqueadas o balanço negativo máximo ocorreu próximo do T165 (Figura 3).

A polpa de araticum in natura apresentou elevado teor de sólidos solúveis, carotenoides e valor de vitamina $\mathrm{A}$.

A pasteurização e o branqueamento não afetaram as características físico-químicas das polpas e reduziram de forma similar o teor de carotenoides e valor de vitamina $A$.

Ambas as temperaturas de congelamento não afetaram as características físico-químicas das polpas e influenciaram o teor de carotenoides de forma semelhante. A variação dos carotenoides e valor de vitamina A nas polpas branqueadas e pasteurizadas submetidas ao congelamento foi similar até 90 dias de armazenamento. Após 180 dias o teor de carotenoides e valor de vitamina A reduziu nas polpas branqueadas/congeladas e aumentou nas polpas pasteurizadas/congeladas.

Assim, recomenda-se o uso do branqueamento/congelamento $-5^{\circ} \mathrm{C}$ para o armazenamento doméstico de polpa de araticum, por até 90 dias e, após esse período, o uso da pasteurização/ congelamento a $-5{ }^{\circ} \mathrm{C}$. Para o produto industrializado, recomenda-se o uso da pasteurização como método de conservação da polpa e armazenamento por até 180 dias. 


\section{ABSTRACT \\ INFLUENCES OF THE BLEACHING, PASTEURIZATION, AND FREEZING ON THE PHYSICOCHEMICAL CHARACTERISTICS AND CAROTENOIDS OF ARATICUM PULP}

This study evaluated the effects of the bleaching, pasteurization, and freezing on the physicochemical characteristics and carotenoids of araticum pulp. The physicochemical characteristics $(\mathrm{pH}$, titratable acidity and soluble solids) and pro- vitamin A carotenoids ( $\alpha$-carotene and $\beta$-carotene) were determined in fresh pulp (control) and in bleached pulp ( $70^{\circ} \mathrm{C}, 2$ minutes) and pasteurized pulp $\left(75^{\circ} \mathrm{C}, 30\right.$ minutes) subjected to the freezing for 180 days $\left(-5\right.$ or $\left.-18{ }^{\circ} \mathrm{C}\right)$. The titratable acidity was determined by volumetric neutralization, $\mathrm{pH}$ by potentiometric and soluble solids by refractometry. Carotenoids were analyzed by high performance liquid chromatography with diode array detection. The fresh pulp showed high levels of soluble solids (21.27 $\left.{ }^{\circ} \mathrm{Brix}\right)$, all-trans- $\alpha$-carotene $(1.98 \mathrm{mg} / 100 \mathrm{~g})$, all-trans- $\beta$-carotene $(1.58 \mathrm{mg} / 100 \mathrm{~g})$ and vitamin A value $(131.46 \mathrm{RAE} / 100 \mathrm{~g})$. The physicochemical characteristics of the pulp remained unchanged after pasteurization and bleaching, and during the storage $(p>0.05)$. However, all-trans- $\alpha$-carotene, all-trans- $\beta$-carotene and vitamin $A$ value decreased after the thermal treatments $(p<0.05)$ (average of $-18,-41$ and $-41 \%$ respectively). During the storage, both the freezing temperatures influenced the pulps in similar manner $(p>0.05)$. Carotenoids and vitamin A value of the bleached or pasteurized frozen pulps similarly ranged up to 90 days of storage. However, after 180 days, the carotenoids and vitamin A value increased in pasteurized pulps (on average, $+6.5 \%,+33.0 \%$ and $+33.5 \%$, respectively) and decreased in bleached pulps $(-23.5 \%,-19.5 \%$ and $-19.5 \%$, respectively). In conclusion, the binomial bleaching/ freezing was effective for storage of pulp up to 90 days and, after that period it is recommended the use of pasteurization/freezing.

PALAVRAS-CHAVE: ANNONA CRASSIFLORA MART.; CAROTENOIDS STABITIY; CERRADO FRUITS; FRUIT PULP.

\section{REFERENCIAS}

1 AMBRÓSIO, C. L. B. et al. Carotenóides como alternativa contra a hipovitaminose A. Revista de Nutrição [S.I.], v. 19, p. 233-243, 2006.

2 BRASIL. Resolução Normativa n 12/78. Aprova Normas Técnicas Especiais do Estado de São Paulo, relativa a alimentos e bebidas. . Brasília: Diário Oficial [da] República Federativa do Brasil, 1978. Instrução Normativa MAA. Regulamento técnico general para fixação dos padrões de identidade e qualidade para polpa de fruta. . Brasília: Diário Oficial [da] República Federativa do Brasil, 2000.

4 Resolução RDC $n^{\circ}$ 12. Regulamento Técnico sobre padrões microbiológicos para alimentos. Brasília, DF: Diário Oficial [da] República Federativa do Brasil, 2001.

5 CARDOSO, L. D. M. et al. Araticum (Annona crassiflora Mart..) from the Brazilian Cerrado: chemical composition and bioactive compounds. Fruits [S.I.], v. 63, n. 2, p. 121-134, 2013.

6 DAMIANI, C. et al. Changes in the physicochemical and microbiological properties of frozen araça pulp during storage. Food Science and Technology (Campinas) [S.I.], v. 33, p. 19-27, 2013.

7 DE SOUZA, V. R. et al. Determination of bioactive compounds, antioxidant activity and chemical composition of Cerrado Brazilian fruits. Food Chemistry [S.I.], v. 134, n. 1, p. 381-386, 9/1/ 2012.

8 ELEZ-MARTÍNEZ, P.; MARTÍN-BELLOSO, O. Effects of high intensity pulsed electric field processing conditions on vitamin $\mathrm{C}$ and antioxidant capacity of orange juice and gazpacho, a cold vegetable soup. Food Chemistry [S.I.], v. 102, n. 1, p. 201-209, 2007.

9 FELLOWS, P. J. Tecnologia do processamento de alimentos: principios e prática. Porto Alegre: ARTMED, 2008.

10 INSTITUTO ADOLFO LUTZ. Normas analíticas do Instituto Adolfo Lutz. São Paulo: Instituto, 2005.

11 KIMURA, M.; RODRIGUEZ-AMAYA, D. B. A scheme for obtaining standards and HPLC quantification of leafy vegetable carotenoids. Food Chemistry [S.I.], v. 78, n. 3, p. 389-398, 2002.

KRINSKY, N. I.; JOHNSON, E. J. Carotenoid actions and their relation to health and disease. Molecular Aspects of 
Medicine [S.I.], v. 26, n. 6, p. 459-516, 2005.

13 LIMA, R. M. T. et al. Estabilidade química, físico-química e microbiológica de polpas de acerola pasteurizadas e nãopasteurizadas de cultivo orgânico. Ciência Rural [S.I.], p. 0-0, 2012.

14 LIN, C. H.; CHEN, B. H. Stability of carotenoids in tomato juice during storage. Food Chemistry [S.I.], v. 90, n. 4, p. 837-846, 5// 2005.

15 MURSU, J. et al. Intake of flavonoids and risk of cancer in Finnish men: The Kuopio Ischaemic Heart Disease Risk Factor Study. International Journal of Cancer [S.I.], v. 123, n. 3, p. 660-663, 2008.

16 OLIVEIRA, K. A. D. M. et al. Desenvolvimento de formulação de iogurte de araticum e estudo da aceitação sensorial. Alimentos e Nutrição [S.I.], v. 19, n. 3, p. 277-281, 2008.

17 PÉNICAUD, C. et al. Degradation of $\beta$-carotene during fruit and vegetable processing or storage: reaction mechanisms and kinetic aspects: a review. Fruits [S.I.], v. 66, n. 06, p. 417-440, 2011.

18 PINHEIRO-SANT'ANA, H. M. et al. Carotenoid retention and vitamin A value in carrot (Daucus carota L.) prepared by food service. Food Chem [S.I.], v. 61, n. 1-2, p. 145-151, 1998.

19 RAMOS, A. M. et al. Manual de boas práticas de fabricação (BPF) para indústrias processadoras de polpa de frutas. Visconde do Rio Branco-MG: Suprema Gráfica e Editora, 2010.

20 RIVAS, A. et al. Effect of PEF and heat pasteurization on the physical-chemical characteristics of blended orange and carrot juice. LWT-Food Science and Technology [S.I.], v. 39, n. 10, p. 1163-1170, 2006.

21 ROCHA, C. et al. Elaboração e avaliação de iogurte sabor frutos do cerrado. B.CEPPA [S.I.], v. 26, n. 2, p. 255-266, 2008.

22 RODRIGUEZ-AMAYA, D. B. A guide to carotenoid analysis in foods. 6. ed. Washington: International Life Sciences Institute, 1999.

23 RODRIGUEZ-AMAYA, D. B.; KIMURA, M. HaverstPlus handbook for carotenoid analysis. Washington: International Food Policy Resarch Institute, 2004.

24 SILVA, D. B. et al. Frutas do cerrado. Brasília, DF: Embrapa Informação Tecnológica, 2001.

25 SOARES, F. P. et al. Marolo: uma frutífera nativa do Cerrado. Lavras/MG: UFLA, 2009.

26 STAHL, W.; SIES, H. Carotenoids and Protection against Solar UV Radiation. Skin Pharmacology and Physiology [S.I.], v. 15, n. 5, p. 291-296, 2002.

27 TAPIERO, H. et al. The role of carotenoids in the prevention of human pathologies. Biomedecine \& Pharmacotherapy [S.I.], v. 58, n. 2, p. 100-110, 2004.

28 U. S INSTITUTE OF MEDICINE. Dietary Reference Intakes (DRIs): Vitamin A, Vitamin K, Arsenic, Boron, Cromium, Copper, lodine, Iron, Manganese, Molybdenium, Nickel, Silicon, Vanadium and Zinc. Washington, D.C.: National Academy Press, 2001.

29 WILliAMS, A. W. et al. Alfa-Carotene Modulates Human Prostate Cancer Cell Growth and May Undergo Intracellular Metabolism to Retinol. The Journal of Nutrition [S.I.], v. 130, n. 4, p. 728-732, 2000.

30 ZEPKA, L. Q. et al. Thermal Degradation Kinetics of Carotenoids in a Cashew Apple Juice Model and Its Impact on the System Color. J Agric Food Chem [S.I.], v. 57, n. 17, p. 7841-7845, 2009/09/09 2009.

\section{AGRADECIMENTOS}

Os autores agradecem à Fundação de Amparo à Pesquisa de Minas Gerais (FAPEMIG), Brasil, pela concessão da bolsa de iniciação científica e apoio financeiro. 